\title{
Una mirada a la evaluación de los aprendizajes en la División de Educación Básica del Centro de Investigación y Docencia en Educación desde el Modelo pedagógico de la Universidad Nacional, Costa Rica
}

\author{
An Analysis of Learning Evaluation in the Basic Education Division of the Center for \\ Research and Teaching in Education based on the Pedagogic Model of the \\ Universidad Nacional, Costa Rica
}

\author{
lleana Castillo Cedeño \\ Decanato del Centro de Investigación y Docencia en Educación \\ Universidad Nacional \\ Heredia, Costa Rica \\ ileana.castillo.cedeno@una.cr
}

Rocío Castillo Cedeño División de Educación Básica, Centro de Investigación y Docencia en Educación Universidad Nacional Heredia, Costa Rica rociocastillocedeno@gmail.com

Luz Emilia Flores Davis ${ }^{3}$ División de Educación Básica, Centro de Investigación y Docencia en Educación Universidad Nacional Heredia, Costa Rica luzdeflores@yahoo.com

Rafael Esteban Jiménez Corrales 4 División de Educación Básica, Centro de Investigación y Docencia en Educación Universidad Nacional Heredia, Costa Rica rejc21@yahoo.com

\footnotetext{
Doctora en Educación con mención en Mediación Pedagógica. Egresada del Doctorado en Diseño Curricular y Evaluación Educativa, Universidad de Valladolid, España. Magíster en Psicopedagogía. Magíster en Educación Preescolar. Especialista en didáctica de la educación del consumidor, Universidad de Valladolid, España. Profesora, bachiller y licenciada en ciencias de la Educación con énfasis en Educación Preescolar, Universidad Nacional. Actualmente se desempeña como investigadora y ocupa el cargo de Decana del Centro de Investigación y Docencia en Educación de la Universidad Nacional de Costa Rica. Autora de varias publicaciones sobre educación, pedagogía y universidad en revistas de su especialidad.

2 Doctora en Educación con mención en Mediación Pedagógica. Magíster en Administración Educativa y en Psicopedagogía. Profesora y bachiller en ciencias de la Educación con énfasis en Educación Preescolar, Universidad Nacional. Veintiún años de experiencia en el ámbito de la educación preescolar y la universitaria. Ha participado activamente en procesos de formación de formadores, principalmente, en el diseño e implementación de cursos de pedagogía a distancia, en la Universidad Estatal a Distancia. Durante cinco años se desempeñó como decana de la Facultad de Educación de la Universidad De La Salle, Costa Rica, y trabajó durante un año como vicerrectora académica en esta misma universidad. Actualmente, se desempeña como docente e investigadora en el Centro de Investigación y Docencia en Educación de la Universidad Nacional de Costa Rica. Autora del libro: La conciencia cósmica en la primera infancia. Reflexiones paradigmáticas, propuestas biopedagógicas y un acercamiento a la espiritualidad de las niñas y los niños.

3 Doctora en Educación con mención en Mediación Pedagógica. Máster en Educación Superior. Catedrática universitaria. Ha ocupado los cargos de directora de Docencia, directora de Desarrollo Profesional y Cooperación Universitaria, presidenta de la Junta de Becas y coordinadora de la Comisión Técnica de Admisión de la Universidad Nacional. Actualmente, se desempeña como investigadora, extensionista y docente en la División de Educación Básica del Centro de Investigación y Docencia en Educación (CIDE) de la Universidad Nacional, Costa Rica y docente en la Maestría en Danza con énfasis en Formación Dancística del Centro de Investigación, Docencia y Extensión Artística (CIDEA), Universidad Nacional, Costa Rica.

4 Doctor en Educación con mención en Mediación Pedagógica. Máster en Evaluación Educativa y Licenciado en Historia. Ha ocupado cargos de subdirector de la División de Educación Básica del Centro de Investigación y Docencia en Educación (CIDE) de la Universidad Nacional, Costa Rica y coordinador de la Maestría en Pedagogía con énfasis en Diversidad en los procesos Educativos. Además, formó parte del equipo de Diseño Curricular de la Dirección de Docencia en esa misma institución. Actualmente, se desempeña como docente, investigador en la División de Educación Básica del Centro de Investigación y Docencia en Educación (CIDE) de la Universidad Nacional, Costa Rica.
} 
URL: http://www.una.ac.cr/educare

CORREO: educare@una.cr

Jorge León Sánchez ${ }^{5}$

División de Educación Básica, Centro de Investigación y Docencia en Educación

Universidad Nacional

Heredia, Costa Rica

jleon@una.cr

Recibido 14 de junio de 2013 • Corregido 30 de julio de 2013 • Aceptado 31 de julio de 2013

Resumen. Los resultados de la investigación que compartimos en el presente artículo centra su mirada en la evaluación de los aprendizajes. El tema seleccionado fue: Operacionalización del modelo pedagógico universitario: Una mirada desde la evaluación de los aprendizajes en cuatro carreras de la División de Educación Básica (DEB) del Centro de Investigación y Docencia en Educación (CIDE) de la Universidad Nacional de Costa Rica (UNA). En dicha investigación se analizaron los principios, estrategias e instrumentos que se aplican en la DEB para evaluar los aprendizajes, con el fin de contribuir a la reflexión y generación de espacios evaluativos más coherentes con lo planteado en el Modelo pedagógico. Producto de la recolección de información mediante el diseño de matrices y la aplicación de cuestionarios dirigidos a docentes y estudiantes, así como los estudios focales, se llega al análisis de los resultados que evidencian la necesidad de trabajar coordinadamente en toda la Universidad para visibilizar y concretizar el Modelo pedagógico de la UNA en lo concerniente a la evaluación de los aprendizajes.

Palabras claves. Evaluación, aprendizaje, pedagogía, educación superior, modelo pedagógico.

Abstract. The research, on which this paper is based, is focused on learning evaluation. The topic selected was: "Operationalization of the University's Pedagogic Model: An analysis of learning evaluation in four study programs of the Basic Education Division (DEB ) of the Center for Research and Teaching in Education (CIDE), at Universidad Nacional (UNA), Costa Rica". This research includes an analysis of principles, strategies, and instruments used in the DEB to evaluate learning, with the purpose of contributing to generate evaluation methods that are more coherent with the Pedagogic Model. The information was collected through focal studies and questionnaires responded by teachers and students; the results reported a need of coordination throughout the entire University to fulfill UNA's Pedagogic Model in terms of learning evaluation.

Keywords. Evaluation, Learning, Pedagogy, Higher Education, Pedagogy Model.

5 Máster en Pedagogía con Énfasis en Atención a la Diversidad, Licenciado en Psicología, ambas por la Universidad Nacional de Costa Rica. Exmiembro del Consejo Universitario de la Universidad Nacional y del Consejo Superior Universitario Centroamericano (CSUCA). Tiene experiencia en atención psicológica para la promoción de la salud y el desarrollo humano integral, así como en programas para el desarrollo del talento y altas capacidades en instituciones educativas. Actualmente, se desempeña como docente e investigador de la División de Educación Básica y la División de Educación Rural del Centro de Investigación y Docencia en Educación (CIDE) de la Universidad Nacional de Costa Rica, y forma parte del Proyecto: Perfiles, dinámicas y desafíos de la educación costarricense. 
La investigación llevada a cabo: “Operacionalización del modelo pedagógico universitario: una mirada desde la evaluación de los aprendizajes en las cuatro carreras de la División de Educación Básica del Centro de Investigación y Docencia en Educación (CIDE) de la Universidad Nacional de Costa Rica" (I. Castillo, R. Castillo, Flores, Jiménez y León, 2013) se realiza en el marco de la implementación del Modelo pedagógico de la Universidad Nacional de Costa Rica (UNA, 2008), entendido como un documento que:

... expresa los principios y lineamientos que orientan el quehacer académico universitario. Es el producto de una construcción participativa y continua, con la que toda la comunidad universitaria debe estar comprometida. Su conocimiento y adopción debe materializarse de manera concreta en la dinámica cotidiana de la institución y de quienes conviven en ella. Como modelo, se deben asumir sus orientaciones de forma general, por ello, se espera que de él deriven estrategias de enseñanza y aprendizaje que se apliquen de manera dinámica, respetando la diversidad en las prácticas pedagógicas y de los objetos de estudio. (Universidad Nacional, 2008, p. 1)

El análisis del modelo indica con toda claridad que se siguieron, para su formulación, las categorías conceptuales creadas por Flórez (1999). En estas se articula la acción pedagógica con respecto a las siguientes categorías:

- Concepto de ser humano y tipo de sociedad.

- El quehacer universitario.

- Los procesos de enseñanza y aprendizaje: concepción (para qué y por qué), el papel de los contenidos (qué), las estrategias mediadoras en el proceso educativo (cómo y cuándo), y las condiciones necesarias para su puesta en práctica.

- Relaciones en el proceso educativo: papel del cuerpo docente, papel del estudiantado y sus responsabilidades.

- La función y la concepción de la evaluación.

- Función de las tecnologías como medio que facilita la interacción entre profesorado, estudiantado y contenidos de aprendizaje.

Es importante destacar que el Modelo pedagógico de la Universidad Nacional está fundamentado en otro proyecto desarrollado por algunas de las personas autoras de este artículo. Como parte de la inquietud de dar seguimiento al proyecto anterior y específicamente a lo que sucedía con la implementación del Modelo pedagógico, se diseñó y desarrolló esta 
URL: http://www.una.ac.cr/educare

CORREO: educare@una.cr

segunda investigación, en la cual se examinaron algunas prácticas evaluativas de las carreras de Bachillerato en Pedagogía con énfasis en I y II ciclos, Pedagogía con énfasis en Educación Preescolar, Educación Especial con énfasis en Integración y Enseñanza del Inglés para I y Il ciclos.

Es relevante mencionar que, además del equipo de investigación, participaron, en las diferentes etapas, estudiantes de las carreras analizadas de la DEB y académicas y académicos de esta división, quienes habían tenido la experiencia de trabajar con el Modelo pedagógico de la UNA.

Valorar el impacto del discurso y la teoría del Modelo pedagógico de la Universidad Nacional de Costa Rica en las acciones concretas de la formación universitaria desde su diseño, en el 2008, se considera un aporte relevante para la UNA, porque permite contrastar la praxis: acción y reflexión sobre la realidad.

Como investigadoras e investigadores se tenía conciencia del compromiso de profundizar en la reflexión y aportar al conocimiento sobre lo que había estado sucediendo con el Modelo, específicamente, en el ámbito de la evaluación de los aprendizajes, reconociendo que el Centro de Investigación y Docencia en Educación en Educación debe convertirse en un referente que aporte a los procesos pedagógicos de toda la Universidad.

En la investigación, se utilizó el cuestionario de preguntas mixtas dirigidas a académicos y académicas, y los focos grupales dirigidos al estudiantado, con el objetivo de triangular la información y poder aportar a la reflexión y generación de espacios evaluativos más coherentes con lo planteado en el Modelo pedagógico de la Universidad Nacional.

\section{Algunos referentes teóricos relevantes para la comprensión de la evaluación de los aprendizajes}

Históricamente y desde un paradigma mecanicista, como elemento del currículo, la evaluación se ha legitimado valorativamente, como la que permite dar fe de los productos o logros alcanzados por el estudiantado en lo cognitivo. Esto ha justificado, desde el nivel inicial hasta el universitario, la evaluación como producto del proceso pedagógico y, por lo general, pese a los discursos de una evaluación democrática, esta tiene un carácter legitimador de las relaciones de poder y control entre los diferentes actores del proceso educativo.

Las investigaciones en torno a la evaluación de los aprendizajes señalan que es necesario desarrollar en el estudiantado capacidades que le permitan su autoevaluación y con ello, regular sus propios procesos de aprendizaje. Una participación activa, crítica y propositiva en el transcurso evaluativo, por parte del colectivo que se ve inmerso en los procesos educativos, favorece la visualización de los cambios en la cultura educativa.

Las sociedades actuales exigen precisamente que sus profesionales, graduados y graduadas de universidades tengan la competencia de valorar su propio proceso de aprendizaje. Esa autonomía hace, que una vez en su desempeño, puedan identificar sus fortalezas y áreas por mejorar en función de una participación ciudadana crítica y ética.

92 Ileana Castillo Cedeño, Rocío Castillo Cedeño,Luz Emilia Flores Davis, Rafael Esteban Jiménez Corrales y Jorge León Sánchez 
Lo anterior demanda que tanto estudiantado como académicos y académicas, además de la institución, conduzcan sus esfuerzos en generar un tipo de cultura evaluativa que, tal y como lo afirman Rodríguez-Gómez, Ibarra-Sáiz, Gallego-Noche, Gómez-Ruiz, y Quesada-Serra (2012), se convierta, en sí misma, quehacer del aprendizaje auténtico. Esto se relaciona con lo planteado por Carless, Joughin y Mok (2006), quienes advierten que la evaluación debe estar orientada al aprendizaje mediante estrategias variadas que favorezcan la autoevaluación, la coevaluación y la evaluación consensuada donde participa el personal docente. Implica un proceso de realimentación y coalimentación que fortalece y mejora la calidad curricular.

Desde esta perspectiva de transformación, es necesario que tanto académicos como académicas se enfrenten al reto de revisar sus propias evaluaciones, las decisiones que toman en torno a estas, los diferentes momentos y componentes que las constituyen, de forma que realmente se promueva una pedagogía que respeta la diversidad en todos sus matices. El diseño, la planificación y el desarrollo de procedimientos de orden evaluativo con participación democrática otorga beneficios relevantes en el compromiso que asume el estudiantado.

Educar en la evaluación es un reto que deben asumir las universidades, de cara a que la evaluación constituye la oportunidad de aprender. Las inconsistencias permanentes y los conflictos que se derivan de prácticas evaluativas son, en gran medida, producto del desconocimiento por parte del profesorado universitario de diferentes estrategias, métodos, técnicas e instrumentos, que en su diseño ofrezcan información trascendente y permitan la toma de decisiones acordes con el contexto sociohistórico y cultural en que se desarrolla el proceso de aprendizaje.

Implica que la Universidad debe redoblar esfuerzos para que cada unidad académica pase del discurso de un modelo pedagógico a la apropiación de este, de acuerdo con su realidad dinámica y al ofrecimiento de oportunidades de formación de las profesionales y los profesionales. Ello conlleva comprender la evaluación como proceso de concertación, de dinamismo metacognitivo que involucra el impulso de procesos de evaluación alternativos, inclusivos. Por lo tanto, entra en juego lo cualitativo y lo cuantitativo, lo individual y lo colectivo, lo racional y lo afectivo; solamente de esta manera se logra optimizar el proceso de aprendizaje, comprendiendo la dimensión formativa de la evaluación; -la evaluación también debe ser considerada como tarea de aprendizaje-.

La evaluación, como tarea formativa, necesita de criterios claros, explícitos por parte del personal docente, que permitan que el estudiantado comprenda y se implique en el proceso pedagógico. Al fin, la evaluación se entiende como un proceso colaborativo que permite el inter-aprendizaje a la luz de la reflexión permanente, lo cual otorga mayor autonomía y confianza a los actores del proceso educativo. Tal y como lo aseguran Álvarez, Padilla, Rodríguez, Torres y Suárez (2011):

La evaluación compartida implica un verdadero trabajo de colaboración entre profesores y estudiantes, que a través de la negociación llegan a establecer criterios y estándares de evaluación, y supone un enriquecimiento mutuo al incrementar el conocimiento sobre el objeto evaluador, en este caso el aprendizaje. (p. 404) 
URL: http://www.una.ac.cr/educare

CORREO: educare@una.cr

Como se mencionó anteriormente, se requiere de una cultura evaluativa que permita, al estudiantado y profesorado, sentirse capaces y cómodos al emitir criterios y valoraciones de sus saberes teóricos, procedimentales y actitudinales; este cambio amerita que se presenten una serie de condiciones que garanticen que la evaluación desarrolle aprendizajes.

\section{Propósitos de la investigación}

El equipo de investigadoras e investigadores se planteó los siguientes propósitos para orientar su búsqueda:

1. Determinar cuáles son los principios en la evaluación de los aprendizajes que sustentan las propuestas generadas en los cursos de cuatro carreras de la División de Educación Básica (DEB) del Centro de Investigación y Docencia en Educación (CIDE), a partir de los planes de estudio y programas de cursos.

2. Analizar los principios en la evaluación de los aprendizajes que sustentan las propuestas generadas en los cursos de cuatro carreras de la DEB del CIDE en el marco del Modelo pedagógico de la Universidad Nacional.

3. Determinar las estrategias utilizadas en la evaluación de los aprendizajes, generadas en los cursos de cuatro carreras de la DEB del CIDE.

4. Analizar las estrategias utilizadas en la evaluación de los aprendizajes que se generan en los cursos de cuatro carreras de la DEB del CIDE en el marco del Modelo pedagógico de la Universidad Nacional.

5. Determinar los instrumentos en la evaluación de los aprendizajes aplicados en los cursos de cuatro carreras de la DEB del CIDE.

6. Analizar los instrumentos utilizados en la evaluación de los aprendizajes que se aplican en los cursos de cuatro carreras de la DEB del CIDE en el marco del Modelo pedagógico de la Universidad Nacional.

7. Generar una propuesta de formación en servicio que fortalezca o cree una práctica evaluativa en los académicos y académicas de la UNA, congruente con el Modelo pedagógico sostenido por esta.

Cabe destacar que los seis primeros propósitos mencionados alimentan el presente artículo.

\section{Proceso metodológico}

Para el abordaje de los propósitos indicados anteriormente, el equipo procedió a identificar los principios evaluativos propuestos en el Modelo pedagógico de la UNA.

Una vez realizado el análisis de los principios de evaluación de los aprendizajes que se encuentran presentes en el Modelo, se concluye que no solamente estos principios están 
en el apartado correspondiente a la evaluación de los aprendizajes, sino que se encuentran diferentes elementos evaluativos en todo el documento. Este propone una evaluación integral, contextualizada, que valora procesos y productos, además de mirar los ambientes de aprendizajes, y concebir al académico o académica como una persona aprendiente más en el proceso de mediación pedagógica. Además, el estudiantado aprendiente es llamado a ser valorado en su integridad como persona.

Seguidamente, se crea una matriz analítica basada en los principios evaluativos de los aprendizajes presentes en el Modelo pedagógico de la UNA. Con ella se valora la presencia de dichos principios en los planes de estudio de las carreras de Bachillerato de la División de Educación Básica a saber: Pedagogía con énfasis en I y II ciclos, Pedagogía con énfasis en Educación Preescolar, Educación Especial con énfasis en Integración y Enseñanza del Inglés para I y II ciclos. La información se presenta en matrices analíticas, se consideran los planes por carrera, luego se elabora una matriz explicativa de síntesis donde se valora la presencia o ausencia de los principios evaluativos del Modelo pedagógico de la UNA. Se concluye que cerca de un $45 \%$ de los principios del modelo evaluativo de la UNA se encuentran presentes en los planes de estudio de las carreras de bachillerato, cerca de un 55\% de dichos principios se encuentran ausentes. Cabe señalar que el comportamiento de los planes en lo que se refiere a la evaluación de los aprendizajes sigue una línea similar.

En un segundo momento, se construye un cuestionario que se aplica a las docentes y los docentes que desarrollaron dichos programas, para valorar su visión y formas de aplicación de los principios de evaluación de los aprendizajes en los cursos. De igual forma, la información se procesa tanto por carrera como de manera general. Se concluye que los programas de curso en su narrativa develan ausencias y presencias de los principios evaluativos del Modelo pedagógico de la UNA, aunque la tendencia es más fuerte hacia la incorporación de ellos. En cuanto a la visión de las académicas y los académicos, la mayoría considera que sí los incorpora en su acción docente, aunque se notan ciertas borrosidades en cuanto a sus concepciones.

Posteriormente, para la detección, descripción y análisis de las estrategias y los instrumentos de evaluación de los aprendizajes que se aplican en los cursos de las carreras, desde la mirada del profesorado y del estudiantado, se procede a elaborar cuatro instrumentos: dos cuestionarios y dos estudios focales. Uno, para valorar las estrategias utilizadas desde la perspectiva docente; y otro, desde el punto de vista del estudiantado. Los dos restantes para la valoración de los instrumentos utilizados en la evaluación de los aprendizajes en los cursos, también según docentes y estudiantes. El procesamiento de los datos se lleva a cabo, de igual manera que en el tratamiento de los principios, elaborando matrices analíticas por carrera y de manera general. De este segundo momento, se extraen cinco tópicos para ser trabajados en la siguiente y última etapa, tanto con estudiantes como con académicos y académicas. Los mismos fueron definidos de la siguiente manera: 
URL: http://www.una.ac.cr/educare

CORREO: educare@una.cr

- Evaluación diagnóstica, formativa y sumativa:El carácter de este tipo de evaluaciones es procesual, integral, concertado, permanente, contextualizado y propositivo, su característica fundamental es ser potenciadora de valorar tanto los aprendizajes, como los procesos de enseñanza.

- Autoevaluación, la coevaluación y la evaluación unidireccional: Esta clasificación busca, en esencia, que los procesos evaluativos no sean de dominio enteramente del personal docente. Los procesos evaluativos deben tener coherencia con los procesos de mediación pedagógica, la cual supone participación activa del estudiantado en su proceso de aprendizaje.

- Interacciones sociales: La evaluación de los aprendizajes implica una amplia visión de los procesos de mediación pedagógica. De esa forma, deben involucrarse elementos tales como los ambiente de trabajo (nichos de aprendizaje), las interacciones que se dan en la clase, la disposición que muestra el estudiantado, tales como puntualidad, participación, disposición para el trabajo en equipo, esfuerzo personal, compromiso ético, social y humanístico, entre otros, además de los logros cognoscitivos. En ese sentido, es fundamental que la evaluación de los aprendizajes considere, en los aprendientes, el desarrollo de habilidades y destrezas de tipo integral, que involucren la capacidad de incluir los nuevos conocimientos en la solución de problemas y en la generación de alternativas; así como el desarrollo y modificación de valores y actitudes; además de la capacidad y la oportunidad de reflexionar sobre la práctica.

- Evaluación concertada: Apela a la creación de una conciencia democrática en la comunidad aprehendiente, estudiantado y docentes, entre otros actores participantes en el proceso formativo; razón por la cual, generar procesos de concertación de la evaluación implica procesos de construcción colectiva.

- Habilidades metacognitivas: Los procesos de evaluación de los aprendizajes deben ayudar a que el estudiantado desarrolle una visión crítica, dinámica y flexible de sus procesos de valoración en sus aprendizajes, apelando a la conciencia y las habilidades de carácter metacognitivo.

A la luz de estos tópicos, se diseñaron y desarrollaron tres talleres, dos con estudiantes y otro con académicas y académicos de la DEB. La información obtenida en estos talleres fue de corte cualitativa, porque apeló al diálogo y la experiencia de sus participantes; permitió la creación de una propuesta de programa de curso de formación en servicio para académicas y académicos de la Universidad Nacional, denominado: La evaluación de los aprendizajes desde el Modelo Pedagógico de la Universidad Nacional. 


\section{Análisis general de los datos}

\section{La evaluación de los aprendizajes en los planes de estudio}

Los principios evaluativos de los planes de estudios de bachillerato de las cuatro carreras de la División de Educación Básica del CIDE manifiestan, de forma heterogénea, el seguir los principios evaluativos del Modelo pedagógico de la Universidad Nacional. Es importante señalar que los planes de estudio fueron diseñados con anterioridad a la creación de este Modelo pedagógico.

No obstante, aparecen claramente identificados en las narrativas de todos los planes de estudios los siguientes principios:

- Se visualiza una evaluación procesual.

- Nace como un proceso concertado.

- Es permanente.

- Se promueve una evaluación contextualizada y propositivo.

- Se valoran tanto los aprendizajes como la enseñanza.

- Se promueve la evaluación diagnóstica, formativa y sumativa.

- Se promueve la autoevaluación, coevaluación y evaluación unidireccional.

- Se evalúan los logros cognoscitivos.

- Se promueve la concertación de la evaluación.

De esa forma, de 20 principios extraídos del Modelo, solamente 9 aparecen totalmente explicitados en los planes de estudio, es decir, un $45 \%$. Por otra parte, el $100 \%$ de los planes de estudio no refleja, en su discurso curricular, los siguientes principios extraídos del Modelo pedagógico de la Universidad Nacional:

- Contempla integralidad.

- Es un quehacer coherente con los procesos didácticos llevados a cabo en el trabajo de aula.

- Genera una visión crítica, dinámica y flexible.

- Promueve procesos metacognitivos.

- Contempla los ambientes de trabajo (nichos de aprendizaje).

- Valora las interacciones que se dan en la clase. 
URL: http://www.una.ac.cr/educare

CORREO: educare@una.cr

- Valora la disposición que muestran los estudiantes (puntualidad, participación, disposición para el trabajo en equipo, esfuerzo personal, compromiso ético, social y humanístico, entre otros).

- Contempla la capacidad de integrar los nuevos conocimientos en la solución de problemas y en la generación de alternativas.

- Considera el desarrollo de habilidades y destrezas.

- Valora el desarrollo y modificación de valores y actitudes.

- Genera la oportunidad de reflexionar sobre la práctica.

Es decir, de 20 principios evaluativos extraídos del Modelo pedagógico de la Universidad Nacional, once no son contemplados de manera explícita en la totalidad de los planes de estudios, lo cual corresponde a un $55 \%$ de ellos. Debe señalarse que el principio: genera oportunidad de reflexionar sobre la práctica, es contemplado solamente por dos carreras de la División.

Algunos otros de los principios, tal como es el caso de promover procesos metacognitivos, no se enuncian en la propuesta evaluativa, pero aparecen como aspectos relevantes de los planes, en este caso, como eje curricular. Es interesante señalar que, a pesar de que la evaluación de los aprendizajes es un componente fundamental en un proceso pedagógico y constituye, muchas veces, un elemento de gran interés para el estudiantado, no se desarrolla con mucho detalle en los planes de estudio. Este componente no tiene la misma fuerza que otros elementos curriculares trabajados en los planes de estudio, como lo son las dimensiones, los saberes y la definición de las áreas y ejes.

\section{La evaluación de los aprendizajes en los programas de los cursos}

En lo concerniente a la valoración de la incorporación de los principios del Modelo pedagógico de la UNA en los programas de los cursos de todas las carreras de la División de Educación Básica, ejecutados durante el primer ciclo lectivo 2011, es importante señalar que dicho análisis se realizó tomando en cuenta todos los componentes del programa de cada curso, no únicamente el apartado referido a la evaluación.

Del análisis realizado se desprende que los siguientes ocho principios del Modelo son asumidos en un $100 \%$ en todos los programas revisados: 
- Se visualiza una evaluación procesual.

- Es permanente.

- Se promueve una evaluación contextualizada y propositiva.

- Es un quehacer coherente con los procesos didácticos llevados a cabo en el trabajo de aula.

- Contempla los ambientes de trabajo (nichos de aprendizaje).

- Valora la disposición que muestran los estudiantes (puntualidad, participación, disposición para el trabajo en equipo, esfuerzo personal, compromiso ético, social y humanístico, entre otros).

- Evalúa los logros cognoscitivos.

- Considera el desarrollo de habilidades y destrezas.

Existe un principio que se considera en un $93 \%$ de los 14 programas revisados: La contemplación de la capacidad de integrar los nuevos conocimientos en la solución de problemas y en la generación de alternativas.

En un $86 \%$ de los programas se contemplan dos principios: generar una visión crítica, dinámica y flexible y la oportunidad de reflexionar sobre la práctica. $Y$ en un $71 \%$ de los programas se toma en cuenta la integralidad y la valoración de las interrelaciones que se dan en la clase. Un 64\% considera el promover los procesos metacognitivos.

Los principios que son menos considerados son los siguientes:

Solamente en un 7\% de los instrumentos se promueve la concertación de la evaluación, y en un $14 \%$ de los programas se considera el desarrollo y modificación de valores y actitudes.

La valoración tanto de los aprendizajes como de la enseñanza no es considerada en ninguno de los programas revisados (0\%).

En un $71 \%$ de los programas revisados no se promueve la evaluación diagnóstica, sumativa y formativa, tampoco la autoevaluación y coevaluación. Como puede verse, predomina la evaluación unidireccional.

En la tabla 1 se indican los principios del Modelo pedagógico en el ámbito de la evaluación de aprendizajes, así como los porcentajes en que se encontraron en los programas de cursos analizados. 
URL: http://www.una.ac.cr/educare

CORREO: educare@una.cr

Tabla 1

Valoración en la evaluación de los aprendizajes de los principios del Modelo pedagógico de la UNA en los programas de curso de cuatro carreras de la DEB

\begin{tabular}{|c|c|c|}
\hline \multirow[b]{2}{*}{ Principios del Modelo Pedagógico } & \multicolumn{2}{|c|}{ Porcentaje } \\
\hline & Sí & No \\
\hline Se visualiza una evaluación procesual. & 100 & \\
\hline Contempla integralidad. & 71.4 & 28,6 \\
\hline Es permanente. & 100 & \\
\hline Se promueve una evaluación contextualizada y propositiva. & 100 & \\
\hline Se valoran tanto los aprendizajes como la enseñanza. & & 100.0 \\
\hline Se promueve la evaluación diagnóstica, formativa y sumativa. & 28.6 & 71.4 \\
\hline Es un quehacer coherente con los procesos didácticos llevados a cabo en el trabajo de aula. & 100 & \\
\hline Se promueve la autoevaluación, coevaluación y evaluación unidireccional. & 28.6 & 71.4 \\
\hline Genera una visión crítica, dinámica y flexible. & 85.7 & 14.3 \\
\hline Promueve procesos metacognitivos. & 64.3 & 35.7 \\
\hline Contempla los ambientes de trabajo (nichos de aprendizaje). & 100 & \\
\hline Valora las interacciones que se dan en la clase. & 71.4 & 28.6 \\
\hline $\begin{array}{l}\text { Valora la disposición que muestran los estudiantes (puntualidad, participación, } \\
\text { disposición para el trabajo en equipo, esfuerzo personal, entre otros). }\end{array}$ & 100 & \\
\hline Evalúa los logros cognoscitivos. & 100 & \\
\hline $\begin{array}{l}\text { Contempla la capacidad de integrar los nuevos conocimientos en la solución de } \\
\text { problemas y en la generación de alternativas. }\end{array}$ & 92.9 & 7.1 \\
\hline Considera el desarrollo de habilidades y destrezas. & 100 & \\
\hline Valora el desarrollo y modificación de valores y actitudes. & 28.6 & 71.4 \\
\hline Genera oportunidad de reflexionar sobre la práctica. & 85.7 & 14.3 \\
\hline Se promueve la concertación de la evaluación. & 7.1 & 92.9 \\
\hline
\end{tabular}

\section{La evaluación de los aprendizajes desde el punto de vista del profesorado}

Del análisis de las respuestas ofrecidas por las académicas y los académicos participantes cabe destacar los siguientes hallazgos:

100 Ileana Castillo Cedeño, Rocío Castillo Cedeño,Luz Emilia Flores Davis, Rafael Esteban Jiménez Corrales y Jorge León Sánchez 
- El 100\% de las personas informantes manifiesta tener total claridad de que durante el curso existe coherencia entre las estrategias didácticas que utilizan con respecto a las estrategias evaluativas que desarrollan. Cabe destacar que este es el único aspecto en el que hay total coincidencia de opinión, y constituye un logro importante en el ámbito de la docencia universitaria.

- Un $67 \%$ de las académicas y académicos expresa que toman en cuenta el Modelo pedagógico de la UNA al elaborar su programa de curso y plantear su propuesta evaluativa. Un 33\% expresa que lo hace parcialmente.

- Respecto a los factores que se establecen en el programa y se evalúan en la práctica de la clase, se da una prioridad a los factores cognitivos, seguida por los socioemocionales y, en último término, los factores espirituales del estudiantado.

- Una gran mayoría (91\%) de las personas participantes manifiesta que en su propuesta evaluativa establecen actividades que les permiten valorar, de forma permanente, los cambios y construcciones en los aprendizajes estudiantiles. Asimismo, que al evaluar tienen presente diferentes dimensiones tales como puntualidad, participación, disposición para el trabajo en equipo, esfuerzo personal, compromiso ético, social y humanístico, entre otros.

- El $82 \%$ de las académicas y académicos participantes afirman que al establecer la evaluación del curso plantean su propuesta y sus estudiantes tienen la posibilidad real de modificarla; así como también tienen espacios para valorar los procesos de enseñanza que realizan, y reflexionar sobre las acciones y aprendizajes desarrollados en la práctica. Ese mismo porcentaje coincide en manifestar que, en la propuesta de evaluación del curso, valoran los materiales que proporcionan, el tipo de aula, los equipos técnicos que utilizan y, si las estudiantes y los estudiantes desarrollan habilidades y destrezas. También que tienen presente, en la evaluación, los valores y actitudes iniciales de sus estudiantes y los comparan con los valores y actitudes al final del proceso, a fin de considerar los cambios generados.

- Si bien llama la atención que solamente el 72,7\% de los académicos y académicas afirma que al formular y desarrollar la evaluación del curso toman en cuenta el nivel cognitivo del estudiantado, más preocupante aún es que ese porcentaje se reduce al $54,5 \%$ cuando se pregunta si en el desarrollo de la evaluación en el curso se tiene presente su formación cultural y social. Este tema es especialmente significativo en una institución que tiene como población meta los estudiantes y las estudiantes de grupos socialmente desfavorecidos.

- De las respuestas expresadas, surge como tema que requiere especial atención, la evaluación diagnóstica. El $18 \%$ del profesorado indica que en el curso parcialmente utiliza y plantea la evaluación diagnóstica y un $9 \%$ dice que no la utiliza. Cuando se pregunta si se toma en cuenta la evaluación diagnóstica para generar los procesos de 
URL: http://www.una.ac.cr/educare

CORREO: educare@una.cr

enseñanza y aprendizaje, solamente el 54,5\% enuncia que sí, mientras que el 27,3\% indica que parcialmente, y el $18,2 \%$ no la toma en cuenta.

- Por otra parte, solamente el 73\% afirma que en el curso plantea, de forma sistemática, una evaluación formativa que vaya guiando en torno a la construcción de aprendizajes de sus estudiantes. Ese mismo porcentaje indica que su curso presenta con toda claridad formas de evaluación sumativas que ofrezcan información sobre los productos de aprendizaje, requerimiento fundamental en un proceso pedagógico.

- Coinciden también el 73\% de informantes, al indicar que consideran que en el curso el proceso evaluativo genera en el grupo de estudiantes una visión crítica, dinámica y flexible de sus procesos de aprendizaje, así como que la evaluación que promueven en el curso hace que sus estudiantes tengan la capacidad de reflexionar sobre cómo aprenden y sobre sus dificultades para lograr aprender.

- Respecto al tema de la autoevaluación, la coevaluación y la evaluación unidireccional, el $82 \%$ opina que, en su propuesta evaluativa del curso, el estudiantado posee un espacio para que desarrolle proceso de autoevaluación; porcentaje que baja al 36.4\% cuando se refieren al desarrollo de procesos de coevaluación. Este tema requiere ser analizado en los equipos de académicas y académicos de las carreras, si se busca fomentar el trabajo en equipo o la constitución de comunidades aprendientes.

\section{La evaluación de los aprendizajes desde el punto de vista del estudiantado}

La valoración brindada por el estudiantado participante se realizó a partir del análisis de tres instrumentos: principios, estrategias e instrumentos que sustentaron la evaluación de los aprendizajes en los cursos del nivel de bachillerato y su relación con el Modelo pedagógico de la Universidad Nacional.

En cuanto a los principios de evaluación, las personas participantes consideraron que los siguientes principios estuvieron presentes por encima del $90 \%$.

- Se visualiza una evaluación procesual.

- Contempla integralidad.

- Es permanente.

- Se promueve una evaluación contextualizada y propositiva.

- Se valoran tanto los aprendizajes como la enseñanza.

- Contempla los ambientes de trabajo (nichos de aprendizaje). 
URL: http://www.una.ac.cr/educare

- Evalúa los logros cognoscitivos.

- Contempla la capacidad de integrar los nuevos conocimientos en la solución de problemas y en la generación de alternativas.

- Genera la oportunidad de reflexionar sobre la práctica.

- Se promueve la evaluación formativa.

- Por otra parte, el estudiantado considera que los siguientes principios estuvieron presentes entre el $80 \%$ y el $89 \%$.

- Es un quehacer coherente con los procesos didácticos llevados a cabo en el trabajo de aula.

- Valora el desarrollo y modificación de valores y actitudes.

- Se promueve la evaluación sumativa.

- Genera una visión crítica, dinámica y flexible.

- Promueve procesos metacognitivos.

- Valora las interacciones que se dan en la clase.

- Valora la disposición que muestra el estudiantado (puntualidad, participación, disposición para el trabajo en equipo, esfuerzo personal, compromiso ético, social y humanístico, entre otros).

- Considera el desarrollo de habilidades y destrezas.

Cabe destacar que los principios evaluativos que se ubicaron con menor presencia en los cursos fueron los siguientes:

- Se promueve la autoevaluación y evaluación diagnóstica; lo consideró un 73\% de las personas participantes.

- Se promueve la concertación de la evaluación, respondió un 72\% de las personas consultadas.

- Se promueve la coevaluación prácticamente, la mitad de participantes 55\%. 
URL: http://www.una.ac.cr/educare

CORREO: educare@una.cr

El análisis realizado a la valoración brindada por el estudiantado en el segundo instrumento (estrategias de evaluación) proyectó los siguientes hallazgos:

- Una gran mayoría del estudiantado (92\%) expresa que existieron estrategias en las cuales ellos y ellas como aprendientes y el mediador o la mediadora propiciaron la valoración de los procesos de enseñanza; aunado a lo anterior, el $91 \%$ consideró que las estrategias evaluativas de los cursos permitieron evidenciar los logros cognoscitivos de las personas aprendientes.

- En la experiencia evaluativa de los cursos, el $82 \%$ del estudiantado está de acuerdo en que se presentaron diversidad de estrategias aplicadas en diferentes momentos. Dichas experiencias integraron en un $85 \%$ conocimientos, actitudes, procedimientos y valores y en un $83 \%$ respondieron a las actividades de provocación de aprendizajes que se generaron en el aula o bien en otros espacios como giras, visitas, entre otros.

- Respecto a si las estrategias de evaluación respondieron a la situacionalidad de las personas aprendientes y a las estrategias de aprendizaje, $86 \%$ del estudiantado estuvo de acuerdo. Sin embargo, el porcentaje baja a un $72 \%$ al consultarles si en los cursos se generaron estrategias que partiendo de condiciones auto-bio-gráficas se iban creando de acuerdo con la particularidad del estudiantado y del grupo. En ese sentido, un $75 \%$ valoró que dichas estrategias evaluativas estaban abiertas al cambio según la incertidumbre de las realidades.

- Por otra parte, el $87 \%$ de estudiantes indicaron que en los cursos aparecieron estrategias de evaluación que valoraron los resultados finales de sus procesos de aprendizaje.

- El 80\% del estudiantado expresó que en la evaluación de los cursos las estrategias les llevaron a lograr criticidad sobre sus propios procesos de aprendizaje. A lo anterior se suma un $83 \%$ de las opiniones que consideró que por medio de las estrategias evaluativas presentes en los cursos se pudo mirar el desarrollo de habilidades y destrezas. No obstante, el $72 \%$ exteriorizó que en la propuesta evaluativa de los cursos las estrategias de evaluación les llevaron a pensar en sus propios procesos de aprendizaje, valorar sus manifestaciones emotivas y analizar las formas de construcción de aprendizajes.

- Asimismo, un $68 \%$ concordó con la opinión de que en la dinámica evaluativa de los cursos se utilizaron estrategias que valoraron los esfuerzos personales y los compromisos que logró el grupo de aprendientes en los campos éticos, sociales y humanísticos. Por lo que en un $69 \%$ las estrategias evaluativas permitieron mirar el cambio y sostenimiento de valores y actitudes. 
- Un $66 \%$ de participantes consideró que las estrategias evaluativas del curso valoraron las interacciones y sinergias que se provocaron. En ese sentido, el $71 \%$ del estudiantado apreció que las estrategias evaluativas del curso permitieron valorar las relaciones que se dieron entre aprendientes y aprendiente-mediador o mediadora.

- Con respecto a si las estrategias evaluativas del curso valoraron los espacios físicos y la infraestructura donde se generaron los procesos de aprendizaje, el $61 \%$ estuvo de acuerdo con ello.

- Únicamente el 69\% de informantes expresaron estar totalmente de acuerdo o de acuerdo con que la evaluación de los aprendizajes nació como proceso concertado en donde ellos y ellas como aprendientes pudieron aportar cambios. Un porcentaje similar (67\%) señaló que en los cursos las estrategias evaluativas nacieron como proceso concertado en donde los mediadores o las mediadoras llevaron registro de cambios.

- Un hallazgo relevante hace referencia a la evaluación diagnóstica, ya que solamente el $63 \%$ del estudiantado manifiesta la existencia de estrategias de evaluación al inicio del curso o al inicio del abordaje de cada unidad temática. Lo anterior reafirma el 54,5\% manifestado por las académicas y los académicos en cuanto a tomar en cuenta la evaluación diagnóstica para generar los procesos de enseñanza y aprendizaje.

- Otro aspecto importante por señalar, con los porcentajes disímiles a lo expresado por las académicas y los académicos participantes respecto al tema de la autoevaluación, la coevaluación y la evaluación unidireccional. El $49 \%$ opina que en la propuesta evaluativa del curso las personas aprendientes tuvieron oportunidad de realizar procesos de autoevaluación; porcentaje que sube ligeramente al 55\% cuando se refieren al desarrollo de procesos de coevaluación y se incrementa al $89 \%$ en procesos unidireccionales de evaluación. Como se señaló anteriormente, este es un tema que requiere ser analizado en los equipos de académicas y académicos de las carreras, si se busca fomentar el trabajo en equipo o la constitución de comunidades aprendientes ya que solamente un $60 \%$ manifestó que las estrategias evaluativas recogieron evidencias del trabajo en equipo. Las personas académicas consideran que realizaron trabajos que recopilaban los aprendizajes del estudiantado, mientras que en los grupos focales, el estudiantado manifestó que hacía falta que se tomara en cuenta sus voces a la hora de definir las estrategias evaluativas. 
URL: http://www.una.ac.cr/educare

CORREO: educare@una.cr

Del tercer instrumento correspondiente a los instrumentos de evaluación de los aprendizajes, cabe destacar los siguientes hallazgos:

- Únicamente un enunciado llegó al 90\% y fue precisamente el que consultaba, si en la dinámica de los cursos, los instrumentos de evaluación se orientaron a que las personas aprendientes pudieran reflexionar sobre lo que hacían en sus horas de práctica.

Nueve enunciados se ubicaron entre el $85 \%$ y el $89 \%$, entre los que destacan los siguientes:

- Valoraron de forma integral conocimientos, actitudes, procedimientos y valores, un $88 \%$.

- Valoraron los resultados finales de sus procesos de aprendizaje, un 88\%.

- Respondieron a la situacionalidad del grupo de aprendientes y a las estrategias de aprendizaje, un $87 \%$.

- Respondieron a las actividades de provocación de aprendizajes que se generaron en el aula o bien en otros espacios como giras, visitas, un $87 \%$.

- Permitieron el seguimiento de las construcciones realizadas por las personas aprendientes, un $87 \%$.

- Aplicaron variados instrumentos evaluativos en el tiempo, un $86 \%$.

- Se presentaron para el estudiantado pudiera aportar cambios, un $85 \%$.

- Permitieron evidenciar los logros cognoscitivos del estudiantado, un $85 \%$.

Igualmente, entre el $80 \%$ y el $84 \%$ se ubicaron nueve enunciados.

- Lograron evidenciar la forma en que iban integrando nuevos conocimientos, cómo crean alternativas de solución a problemas y generaban nuevas respuestas, un $84 \%$.

- Valoraron los procesos de aprendizaje, las interacciones y sinergias que se provocaron, un $84 \%$.

- Se utilizó diversidad de instrumentos, un 83\%.

- Permitieron valorar saberes procedimentales por parte de ellos y ellas como aprendientes, un $83 \%$.

106 Ileana Castillo Cedeño, Rocío Castillo Cedeño,Luz Emilia Flores Davis, Rafael Esteban Jiménez Corrales y Jorge León Sánchez 
URL: http://www.una.ac.cr/educare CORREO: educare@una.cr

- Estuvieron abiertos al cambio, según la incertidumbre de las realidades, un $81 \%$.

- Consideraron las condiciones auto-bio-gráficas del estudiantado, un 81\%.

- Llevaron a lograr criticidad sobre sus propios procesos de aprendizaje, un $81 \%$.

- Nacieron como proceso concertado en donde los mediadores o las mediadoras, llevaron registro de cambios, un $80 \%$.

- Presentaron formas que podían recoger evidencias del trabajo en equipo, un $80 \%$.

Por otra parte, entre el $75 \%$ y el $79 \%$ se encontraron cuatro enunciados:

- Permitieron registrar la puntualidad de las aprendientes y los aprendientes, un 79\%.

- Permitieron valorar las relaciones que se dieron entre las aprendientes y los aprendientes, o entre aprendiente-mediador o mediadora, un 77\%.

- Llevaron a pensar en sus propios procesos de aprendizaje, valorar sus manifestaciones emotivas y analizar las formas de construcción de aprendizajes, un 76\%.

- Valoraron los espacios físicos y la infraestructura donde se generaban los procesos de aprendizaje, un $76 \%$.

En cuanto al rango comprendido entre el $70 \%$ y el $74 \%$ se hallaron cinco enunciados:

- Propiciaron la valoración de los procesos de enseñanza por parte de las aprendientes y los aprendientes y el mediador o la mediadora, un $74 \%$.

- Valoraron los esfuerzos personales y los compromisos que lograban las aprendientes y los aprendientes en los campos éticos, sociales y humanísticos, un $74 \%$.

- Permitieron observar el desarrollo de habilidades y destrezas, un $72 \%$.

- Permitieron registrar la participación, un 71\%.

- Permitieron la autoevaluación, un 70\%. 
URL: http://www.una.ac.cr/educare

CORREO: educare@una.cr

Especial atención merecen los enunciados que obtuvieron un porcentaje menor al 70\%, destacándose los siguientes aspectos:

- Permitieron mirar el cambio y sostenimiento de valores y actitudes, un $68 \%$.

- Permitieron la coevaluación, un 64\%.

- Permitieron la evaluación diagnóstica al inicio del curso o al inicio del abordaje de cada unidad temática, un $62 \%$.

- Valoraron los propios procesos del estudiantado $54 \%$.

\section{Algunos aportes y comentarios del personal docente entrevistado}

El Modelo pedagógico es una senda donde se plasman los grandes ideales o metas de formación de las relaciones entre aprendientes en las diferentes ofertas académicas de la Universidad Nacional. Y ese modelo cobra vida en su operacionalización, por lo cual son fundamentales las perspectivas de las académicas y los académicos, así como del estudiantado. Seguidamente, se transcriben, en primera instancia, algunas expresiones y aportes de académicas y académicos participantes en la investigación:

"... toda estrategia planteada evalúa tanto al estudiante como al docente".

“...la auto y co evaluación requieren de cierta concientización previa de parte de los profesores, pues creo que no hemos logrado que las estudiantes tengan un nivel de reflexión necesario para este tipo de evaluaciones. Lo he utilizado en otras oportunidades y creo que no logra lo que pretende".

"...creo que hay niveles como los iniciales que requieren de ciertos formalismos y rigurosidad, acompañado creo yo de una constante concientización sobre el papel y responsabilidad que ellas tendrán desde su profesión como docentes".

"En realidad creo que el tema de las prácticas evaluativas es fundamental para una mejor práctica docente, considero que hay mucho desconocimiento sobre el tema, no sabemos cómo evaluar para potenciar el aprendizaje y la reflexión de las estudiantes sobre lo que aprenden y cómo lo aprenden. Creo que por lo tanto no podemos pretender estudiantes reflexivas que puedan expresar ideas concretas y sólidas, que tengan claridad ante procesos más complejos y sobre todo que no estamos propiciando desde nuestras propuestas evaluativas un cambio en la visión de evaluación que estas futuras docentes manejarán en sus aulas". 
“Yo utilizo mucho la hora de atención a estudiantes para valorar los procesos de cada quien, además en el aula regularmente se van valorando avances u obstáculos presentes. Para mí la evaluación debe incluso ir buscando un modelo emancipador, en donde el estudiante logre comprender que el proceso no está centrado en la nota que pueda otorgar un profesor determinado, sino más bien en una construcción positiva y firme de su propio perfil profesional".

"No se puede ser cortoplacista, algunos aprendizajes que alcanzan nosotros nunca los vamos a percibir de inmediato en un curso sino que aflorarán cuando tengan que aflorar (Howard Gardner)...en las situaciones menos pensadas o estructuradas".

"Tengo pendiente más rigurosidad a la hora de mi autoevaluación, es un proceso que llevo paralelo a mi quehacer, lo pienso pero no lo tengo sistematizado, la tengo más ligada a mi saber hacer, al oficio de enseñar. Creo que con los años he aprendido a "flexibilizar" mis actuaciones y posturas, una cierta adaptación al medio, a los actores y sus circunstancias".

"Me cuestiono el separar los saberes cognitivos, procedimentales y actitudinales, constituye una división analítica del proceso pero no real, las tres dimensiones son interdependientes y es difícil a veces dibujar fronteras entre ellas. No pueden ser más que aprehendidas parcialmente dado lo "irreductible" del proceso de aprendizaje que a veces ni siquiera es totalmente consciente para la persona que lo vive menos para el docente que lo mira "desde afuera". Hago esfuerzos por llegar a ciertas evidencias más "objetivas" pero sé que no reflejan ni lejanamente la complejidad del proceso evaluativo. Con toda honestidad, la evaluación me parece lo más ajeno e impuesto institucionalmente al proceso pedagógico, de hecho no apareció sino hasta finales del siglo XVIII y sin duda ha "enturbiado" el panorama con requerimientos de validación social del conocimiento, de un determinado tipo de conocimiento seleccionado por el curriculum, que poco o nada tienen que ver con la esencia de aprender-compartir. Sé que estamos obligados a tomarlo en cuenta en la Universidad y que esfuerzos como la presente investigación son muy importantes en este sentido, pero que lindo sería no tener que evaluar Sólo permitir que aprendamos sin más, parafraseando al viejo Marx"cada uno según su capacidad y a cada uno según su necesidad". 
URL: http://www.una.ac.cr/educare

CORREO: educare@una.cr

"Muchas gracias por darme la oportunidad de reflexionar sobre las vivencias del curso, lo cual, facilitará el poder mejorar aspectos que se pueden fortalecer".

"Considero que existen procesos evaluativos que no se reflejan necesariamente en el programa presentado, sino más bien que se originan en las interacciones y en la realidad de la clase".

"Hay afirmaciones que me parecen interesantes y que sería muy viable cumplir si a nivel de normativa universitaria existiera mayor flexibilidad en los procesos evaluativos. Se asume que una vez acordados los procedimientos evaluativos, con los y las estudiantes, éstos no sufrirán cambios radicales. Muchas veces la opción que yo asumo es cambiar estrategias, o brindar oportunidades para presentar los trabajos o actividades de otras maneras que mejor respondan a las dinámicas del curso o a las necesidades de los y las estudiantes, pero mentiría si dijera que ello se refleja en cambios en los rubros evaluativos".

\section{Algunos aportes y comentarios del estudiantado de las carreras de la DEB}

Seguidamente, se destacan algunas expresiones y aportes desde la perspectiva estudiantil.

"Considero acerca de la evaluación formativa que es esencial, por lo que como estudiantes en formación requerimos de formación humana y vivenciar lo más cercano posible en lo que deseamos especializarnos, donde se adquiera una empatía en el contexto. Es decir, fortalecer aún más la evaluación de este tipo donde sea mayor el contacto con las personas, más aún en este servicio, vocación como lo es la Educación Especial de forma que no solo se limiten a visitar escuelas sino más bien otros lugares donde brindemos apoyos y servicios que en la misma Universidad se nos enseña, ya sea a empresas, centros penitenciarios, albergues, centros de ayuda al adulto mayor, entre otros".

"Es pertinente y necesario que los profesores realicen una serie de diagnósticos para que puedan comprender las realidades del contexto de muchos alumnos. Me parece que muchas veces el discurso entra en una contradicción pedagógica"

"Considero que se debe tomar más en cuenta el esfuerzo personal, considero que una evaluación debe ser flexible pero no permitir que estudiantes que no demuestran buen desempeño logren seguir avanzando con los mismos beneficios" 
"Valorar un poco más los aspectos no sumativos, tales como: resolución alternativa de conflictos al realizar intervenciones en el curso (trabajos) o en horas práctica; Iniciativa y creatividad; aporte de ideas y sugerencias en clase, actitudes asertivas, entre otras".

"En algunos de los cursos, la evaluación responde al estilo y personalidad de la docente, dejando de lado los intereses y necesidades del estudiantado."

"El conductismo caracteriza la evaluación en donde solo se le da importancia a los resultados así como al formato y no el contenido".

"Considero que en ocasiones las técnicas utilizadas son un poco tradicionales, por ejemplo la entrega semanal o diario de crónicas o reportes de visita. Quizá podría evaluarse el proceso de una forma más funcional como foros o conversatorios o presentaciones con fotografías... donde los avances de cada semana sean evidenciados y pueda darse una retroalimentación con el trabajo del resto de compañeros y compañeras".

"Los estudios de caso son una dinámica muy interesante, además me gustaría que se pudieran construir los instrumentos de evaluación en conjunto".

\section{Aprendizajes relevantes}

A manera de conclusión se subraya la importancia de que la evaluación de los aprendizajes tenga un carácter procesual, integral, concertado, permanente, contextualizado y propositivo, que valore tanto los aprendizajes como la enseñanza.

Se requiere de un proceso evaluativo que cuente al menos con las tres etapas o momentos básicos, a saber: evaluación diagnóstica, formativa y sumativa.

Es necesario que los procesos de evaluación de los aprendizajes sean coherentes con la mediación pedagógica llevada a cabo en el trabajo de aula. Además, buscar que los procesos evaluativos no sean de dominio únicamente del facilitador o facilitadora, debe existir momentos para la autoevaluación, coevaluación y evaluación unidireccional.

Los procesos de evaluación de los aprendizajes deben ayudar a que el estudiantado posea y desarrolle una visión crítica, dinámica y flexible de sus procesos de valoración en sus aprendizajes. De esa forma, se persigue una conciencia y habilidades de carácter metacognitivo en las aprendientes y los aprendientes. 
URL: http://www.una.ac.cr/educare

CORREO: educare@una.cr

La evaluación de los aprendizajes implica una amplia visión de los procesos de mediación pedagógica; por ello, es importante que se involucren elementos tales como los ambientes de trabajo (nichos de aprendizaje), las interacciones que se dan en la clase, la disposición que muestra el estudiantado (puntualidad, participación, disposición para el trabajo en equipo, esfuerzo personal, compromiso ético, social y humanístico, logros cognoscitivos, entre otros.). En ese sentido, es medular que la evaluación de los aprendizajes mire y considere el desarrollo de habilidades y destrezas, que se generan en las aprendientes y los aprendientes.

Finalmente, es urgente que la evaluación de los aprendizajes apele a la creación de una conciencia democrática en las aprendientes y los aprendientes, para lo cual es necesario que se generen procesos de concertación de la evaluación, de manera que sean procesos de construcción colectiva.

En síntesis, los principios de evaluación de los aprendizajes que emanan del Modelo pedagógico de la Universidad Nacional son los siguientes:

- Procesual, integral, concertado, permanente, contextualizado y propositivo.

- Tanto los aprendizajes como la enseñanza son sujetos de evaluación.

- Diagnóstica, formativa y sumativa.

- Coherente con los procesos didácticos llevados a cabo en el trabajo de aula.

- Promotora de procesos de autoevaluación, coevaluación y evaluación unidireccional.

- Poseedora de una visión crítica, dinámica y flexible.

- Promotora de procesos metacognitivos.

- Valoradora de los ambientes de trabajo (nichos de aprendizaje).

- Observadora de las interacciones que se dan en la clase.

- Atenta a valorar la disposición que muestra el estudiantado (puntualidad, participación, disposición para el trabajo en equipo, esfuerzo personal, compromiso ético, social y humanístico, entre otros.

- Valora los logros cognoscitivos.

- Capaz de considerar el desarrollo de habilidades y destrezas.

- Generadora de la capacidad de integrar los nuevos conocimientos en la solución de problemas y en la generación de alternativas.

- Capaz de promover el desarrollo y modificación de valores y actitudes.

- Generadora de oportunidades para reflexionar sobre la práctica.

- Promotora de procesos de concertación de la evaluación misma. 
Con fundamento en lo anterior, el equipo investigador propone los siguientes aspectos a considerar en la normativa de la Universidad Nacional de Costa Rica que orienten las prácticas evaluativas de los cursos:

- Presentar diversidad de estrategias e instrumentos para aplicar en diferentes momentos pero no como cortes, sino siguiendo una lógica de alcance y secuencia, con la idea de generar un corpus evaluativo donde una estrategia se relacione con la otra.

- Integrar la evaluación de conocimientos, actitudes, procedimientos y valores (estrategias que abiertamente incluyan momentos en que se manifiestan el saber hacer, el saber ser, el saber cómo).

- La evaluación de los aprendizajes debe nacer como proceso concertado en donde el estudiantado pueda aportar cambios, lo cual se debe reflejar en una estrategia y momento para ello, donde se indiquen las formas que permite su participación. Por ejemplo: diálogos, bitácora de acuerdos, entre otros.

- Aplicar variadas estrategias evaluativas en el tiempo, estrategias que tengan una lógica entre sí a lo largo de todo el proceso.

- Responder a la situacionalidad del estudiantado, así como a las estrategias de aprendizaje, por lo tanto, es necesario que las estrategias de evaluación y los instrumentos consideren las condiciones auto-bio-gráficas y se establezcan de acuerdo con la particularidad de los estudiantes y las estudiantes y del grupo. Ello implica que estén abiertas al cambio según la incertidumbre de las realidades.

- Incluir estrategias e instrumentos en los cuales las personas aprendientes y el mediador y la mediadora propicien la valoración de los procesos de enseñanza.

- Utilizar estrategias de evaluación y sus respectivos instrumentos, al inicio del curso y al inicio del abordaje de cada unidad temática; así como durante el proceso de manera que permitan el seguimiento de las construcciones realizadas por los aprendientes y las aprendientes. También, hacer uso de estrategias de evaluación e instrumentos que valoren los resultados finales de los procesos de aprendizaje.

- Es fundamental que las estrategias e instrumentos de evaluación respondan a las actividades de provocación de aprendizajes que se generan en el aula o bien en otros espacios como giras, visitas, entre otros.

- Incluir estrategias e instrumentos para que los aprendientes y las aprendientes se autoevalúen, se evalúen entre pares y para que el facilitador o facilitadora les evalúe.

- Las estrategias deben llevar al estudiantado a lograr criticidad sobre sus propios procesos de aprendizaje. Incluso puede aportar estrategias e instrumentos de evaluación de sus propios procesos. 
URL: http://www.una.ac.cr/educare

CORREO: educare@una.cr

- Utilizar estrategias de evaluación e instrumentos que lleven al estudiantado a pensar en sus propios procesos de aprendizaje, a valorar sus manifestaciones emotivas y analizar las formas en que construyen aprendizajes.

- Valorar los lugares físicos donde se generan los procesos de aprendizaje, así como las interacciones y sinergias que esos espacios provocan.

- Valorar las relaciones que se dan entre aprendientes, aprendiente-mediador, aprendientes-currículo, mediador-currículo.

- Registrar la puntualidad y la participación del estudiantado

- Recoger evidencias del trabajo en equipo.

- Utilizar estrategias que valoren los esfuerzos personales y los compromisos que logran los estudiantes y las estudiantes en los campos éticos, sociales y humanísticos.

- Evidenciar los logros cognoscitivos del estudiantado.

- Evidenciar como las personas aprendientes van integrando nuevos conocimientos, como crear alternativas de solución a problemas y generar nuevas.

- Valorar el desarrollo de habilidades y destrezas, es decir, los saberes procedimentales que construye el estudiantado.

- Apreciar el cambio y sostenimiento de valores y actitudes en el estudiantado.

- Promover la reflexión sobre lo que hace el estudiantado en sus horas de práctica o en los procesos generados en ella.

El Modelo pedagógico de la Universidad Nacional requiere de acciones concretas de operacionalización. En toda propuesta pedagógica los principios orientadores e inspiradores deben vivenciarse en la práctica, sino sería insuficiente todo esfuerzo de construcción. Por lo anterior, es importante que los principios enunciados se traduzcan en estrategias evaluativas e instrumentos coherentes con ellos. Se necesita fortalecer la formación permanente para docentes, de manera que se sostengan, refuercen o ejecuten prácticas evaluativas congruentes con el Modelo pedagógico asumido por la Universidad Nacional de Costa Rica en el que se refleja una visión de la evaluación con enfoque socio-crítico.

114 Ileana Castillo Cedeño, Rocío Castillo Cedeño,Luz Emilia Flores Davis, Rafael Esteban Jiménez Corrales y Jorge León Sánchez 


\section{Referencias}

Álvarez, V., Padilla, M. T., Rodríguez, J., Torres, J. J. y Suárez, M. (setiembre-diciembre, 2011). Análisis de la participación del alumnado universitario en la evaluación de su aprendizaje. Revista española de pedagogía, 69(250), 401-425.

Carless, D., Joughin, G. y Mok, M. M. (agosto, 2006). Learning-oriented Assessment: Principles and Practice [La evaluación orientada al aprendizaje: Principios y práctica]. Assessment \& Evaluation in Higher Education, 31(4), 395-398.

Castillo, I., Castillo, R., Flores, L., Jiménez, R. y León, J. (2013). Informe final de Proyecto de Investigación "Operacionalización del modelo pedagógico universitario: Una mirada desde la evaluación de los aprendizajes en las cuatro carreras de la División de Educación Básica del Centro de Investigación y Docencia en Educación (CIDE) de la Universidad Nacional de Costa Rica". Heredia, Costa Rica: Universidad Nacional.

Flórez, R. (1999). Evaluación pedagógica y cognición. Bogotá: McGraw-Hill.

Rodríguez-Gómez, G., Ibarra-Sáiz, M. S., Gallego-Noche, B., Gómez-Ruiz, M.-Á. y Quesada-Serra, V. (2012). La voz del estudiante en la evaluación del aprendizaje: Un camino por recorrer en la universidad. RELIEVE, 18(2), 133-153.

Universidad Nacional. (2008). Modelo pedagógico de la Universidad Nacional. Sistema de gestión y descarga de documentos electrónicos, Vicerrectoría Académica. Recuperado de http:// www.una.ac.cr/index.php?option =com remository\&ltemid=0\&func=startdown\&id $=141$

\section{Cómo citar este artículo en APA:}

Castillo, I., Castillo, R., Flores, L. E., Jiménez, R. E. y León, J. (2013). Una mirada a la evaluación de los aprendizajes en la División de Educación Básica del Centro de Investigación y Docencia en Educación desde el Modelo pedagógico de la Universidad Nacional. Revista Electrónica Educare, 17(3), 89-115. Recuperado de http://www.revistas.una.ac.cr/index.php/EDUCARE/issue/current

Nota: Para citar este artículo en otros sistemas puede consultar el hipervínculo "Como citar el artículo" en la barra derecha de nuestro sitio web:

http://www.revistas.una.ac.cr/index.php/EDUCARE/index 